\title{
Density determination of the thermonuclear fuel region in inertial confinement fusion implosions
}

P. L. Volegov ${ }^{1}$, S. H. Batha ${ }^{1, a)}$, V. Geppert-Kleinrath ${ }^{1}$, C. R. Danly ${ }^{1}$, F. E. Merrill ${ }^{1}$, C. H. Wilde $^{1}$, D. C. Wilson ${ }^{1}$, D. T. Casey ${ }^{2}$, D. Fittinghoff ${ }^{2}$, B. Appelbe ${ }^{3}$, J. P. Chittenden ${ }^{3}$, A. J. Crilly $^{3}$, and K. McGlinchey ${ }^{3}$

\author{
${ }^{1}$ Los Alamos National Laboratory, Los Alamos, New Mexico 87545, United States \\ ${ }^{2}$ Lawrence Livermore National Laboratory, Livermore, California 94550, United States \\ ${ }^{3}$ Centre for Inertial Fusion Studies, The Blackett Laboratory, Imperial College, London \\ SW72AZ, United Kingdom
}

\begin{abstract}
Understanding of thermonuclear burn in an inertial confinement fusion implosion requires knowledge of the local deuterium-tritium (DT) fuel density. Neutron imaging of the core now provides this previously unavailable information. Two types of neutron images are required. The first is an image of the primary $14-\mathrm{MeV}$ neutrons produced by the $\mathrm{D}+\mathrm{T}$ fusion reaction. The second is an image of the $14-\mathrm{MeV}$ neutrons that leave the implosion hot spot and are downscattered to lower energy by elastic and inelastic collisions in the fuel. These neutrons are measured by gating the detector to record the 6-12 MeV neutrons. Using the reconstructed primary image as a nonuniform source, a set of linear equations is derived that describes the contribution of each voxel of the DT fuel region to a pixel in the downscattered image. Using the measured intensity of the $14-\mathrm{MeV}$ neutrons and downscattered images, the set of equations is solved for the density distribution in the fuel region. The method is validated against test problems and simulations of high-yield implosions. The calculated DT density distribution from one experiment is presented.
\end{abstract}

\footnotetext{
a) Author to whom correspondence should be addressed: sbatha@lanl.gov
} 


\section{Introduction}

The hot-spot approach to inertial confinement fusion (ICF) ${ }^{1}$ uses an energetic laser or pulsed-power driver to assemble a quantity of deuterium-tritium (DT) fuel into a hot and dense plasma in a very particular way. The inner fuel region, called the hot spot, is hot but relatively less dense. The outer fuel region, called the cold fuel, is cold but relatively denser. Outside of the fuel is what remains of the ablator material that created the implosion by converting driver energy into internal energy of the fuel. To achieve ignition, the hot spot must reach sufficiently high temperatures and densities ${ }^{2,3}$ for the DT ions to start reacting and producing neutrons and alpha particles. These alpha particles deposit their energy in the cold fuel and heat it to thermonuclear conditions. In this way, the plasma ignites in the hot spot and then forms a propagating burn wave that ignites the cold fuel. ${ }^{3}$

The hot spot and cold fuel are nominally in pressure equilibrium, but have very different temperatures and densities. The size of the fuel region indicates the amount of compression of the fuel. ${ }^{4,5}$ Recent research into the effect of mixing on the energy partition between the hot spot and cold fuel ${ }^{6}$ demonstrates that a more detailed understanding of the state of the cold fuel is needed to comprehend the burn process in near-ignition capsule implosions fully.

The hot-spot temperature is measured using neutron time-of-flight (nToF) methods, ${ }^{7-9}$ and the hot-spot density can be estimated from the total yield and size of the $14 \mathrm{MeV}$ emitting region that is measured by the neutron imaging system. Because the cold fuel does not emit neutrons and the DT ions are fully stripped, neither the neutron nor the spectroscopic information about the cold fuel is available, and only average quantities regarding the cold fuel are measurable. For example, each nToF detector measures the downscattered ratio (DSR), which is the total amount of scattering in the cold fuel along one line of sight of that detector. Additionally, each flange-mounted neutron activation detector (FNAD) ${ }^{10}$ measures the amount of attenuation of neutrons as they pass through the cold fuel, again along one line of sight. While there are several nToF and many FNAD detectors on the National Ignition Facility (NIF), neither set of detectors gives the density distribution of the cold fuel with resolution approaching that of the neutron imaging system (NIS).

To allow cold-fuel density measurement and detailed density mapping of the entire fuel region, downscattered neutron imaging was developed and deployed at the NIF. ${ }^{10-13}$ The work described here demonstrates how the density of the cold fuel can be determined in absolute units to first order through the combined use of the measured primary and downscattered neutron images. Section II describes the neutron imaging system at NIF and its ability to measure the downscattered image. Using the measured primary image, the measured downscattered image, and calculations of the neutron scattering, the cold-fuel density is determined in Section III. The method is validated with computer simulations in Section IV and applied to experimental data in Section V.

\section{The neutron-imaging-system measurement}

The NIS at NIF is continuously evolving. NIS began with a single line of sight (NIS1) observing the implosion from the target chamber equator, which is at 90 degrees from the hohlraum's laser entrance holes, at the target chamber port $(90,315) \cdot{ }^{10-14}$ NIS1 measures 
both the primary 14-MeV neutron image and the downscattered neutron image. The image is formed by an array of apertures machined into 20-centimeter-long gold foils. ${ }^{14,15}$ The apertures have one of two shapes. The 20 pinholes are small triangular grooves machined into the gold. The three mini penumbral apertures have a double conic shape. The location of each aperture was carefully measured after machining and prior to assembly, and then verified during many NIF experiments. ${ }^{14}$

A second system, NIS2, at the pole of the chamber was added to measure the primary image. ${ }^{16,17}$ A third line of sight (NIS3) at the equator currently measures the primary image, and it will soon be upgraded to also measure the downscattered image and the gamma image from interactions of the neutrons with the carbon ablator. The work presented here is based on primary images from all three lines of sight and on the downscattered image obtained from NIS1.

On NIS1, the images formed by the pinhole array are detected by a fiber optic array comprised of 250-micron-diameter by 5-centimeter-long BCF-99-55 scintillating fibers. ${ }^{18}$ The array is imaged by two camera systems, one observing the front of the fiber array and one observing the back. Independently gated microchannel-plate intensifiers that are located between the scintillator and the charge-coupled device (CCD) cameras allow two different energy ranges to be measured. For density measurements, the primary image is gated for 13-17 MeV neutrons. The downscattered image gate is set for 6-12 MeV neutrons. The energy range is wide to measure as many neutrons as possible, but it is limited by the finite rise time of the gated camera system at the high-energy end and by the decreasing scattering cross section at the low-energy end. ${ }^{19}$ Not all of the cold fuel region is sampled by the downscattered image due to the kinematics of the elastic collisions. ${ }^{19}$ About 40 percent of the cold fuel region is sampled in an annular region around the direction of observation. When all three NIS lines of sight are fully instrumented to record the downscattered neutron images, 79 percent of the fuel will be measured, with 28 percent of the fuel measure by two detectors and 8 percent measured by three detectors. ${ }^{19}$

Because neutrons have a long mean free path (about 3 centimeters) in the gold layers of the aperture array, the pinhole does not behave as a thin aperture as it does in a standard optical or x-ray pinhole camera. Instead, the aperture is thick with edges that can be penetrated by the neutron so that the image formed is not spatially shift invariant, which complicates the image reconstruction. ${ }^{20}$

After maximum-likelihood image reconstruction, the estimated spatial resolution is approximately 10 microns in the object plane, and Legendre polynomial fits to the reconstructed image have uncertainties in the coefficients that are estimated to be a few microns. ${ }^{14}$

Typical neutron images from a NIF implosion are shown in Figure 1 for experiment, N190120-001, a high-performance implosion using a high-density carbon (HDC) capsule. $^{21}$ The experiment produced greater than $9 \times 10^{15}$ neutrons. The primary images were recorded along the two equatorial $(90,315$ and 90,213) and the polar $(5,224)$ lines-ofsight. The polar primary image is approximately round with a mean radius of 46 microns and a Fourier-mode $M_{2}$ coefficient of -3 microns at the 17 percent of maximum intensity. Both equatorial primary images are approximately elliptical with mean radii of 30 microns and Legendre-mode $P_{2}$ coefficients of -15 microns. The down scattered image was 
recorded only along the $(90,315)$ equatorial line of sight. The scattered image is larger, with a radius of approximately 48 microns and a $P_{2}$ coefficient of -9 microns.
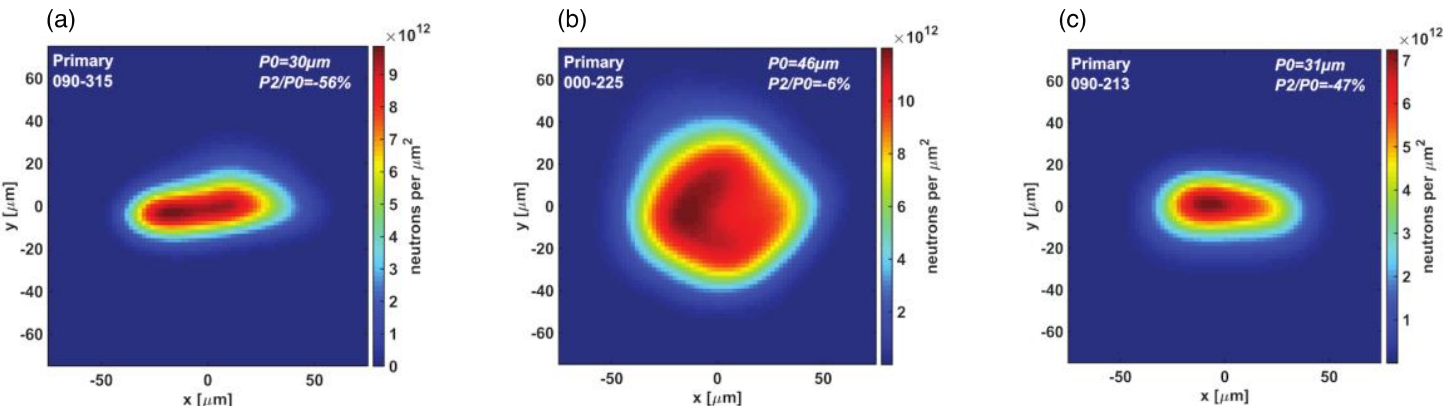

(d)

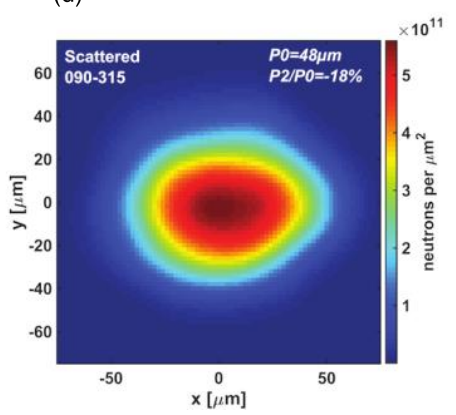

(e)

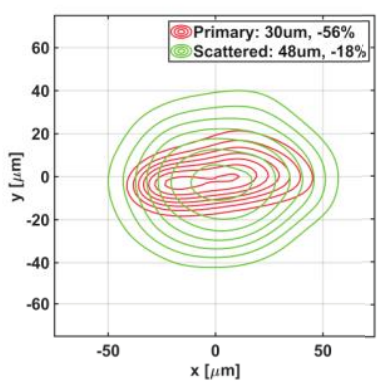

Figure 1. Measured primary neutron images from three lines of sight: (a) NIS1, (b) NIS2, and (c) NIS3 from NIF shot N190120-001. The downscattered image from NIS1 is shown in (d). Isocontours of the number of primary and downscattered neutrons are superimposed in $(e)$.

Registration of primary and downscattered images is determined via a dedicated neutronemitting experiment on the NIF. In this procedure, the pinhole array is removed and a 50mm-thick copper block is placed into the path of the neutrons, about $30 \mathrm{~cm}$ from the scintillator. The copper block has 528 3-mm-diameter holes drilled through it on 6-mm centers in a 23 by 23 grid pattern, with one hole removed to provide an orientation fiducial. The neutrons pass through the holes in the block and create a pattern of bright spots on the scintillator. The spots are viewed simultaneously from front and back and recorded on the primary and downscattered cameras. The two images can then be registered to each other with an accuracy of better than 80 microns in the image plane ensuring that the reconstructed primary and downscattered images are co-registered with an accuracy better than 1 micron in the source plane since the system has a magnification of 85 . The adequacy of this method is discussed in Section V. 


\section{Determination of the fuel density distribution}

The three-dimensional fuel density distribution, $\rho(\mathbf{r})$, is determined from the NIS measurements, the known geometry of the NIS, and the known scattering cross sections. ${ }^{22}$ The downscattered image is formed by $14-\mathrm{MeV}$ neutrons produced by DT reactions in the hot spot of the implosion elastically scattered from ions in the DT region of the plasma. The neutrons can scatter from ions within the hot spot of the implosion as well as in the cold fuel region. The measurement is predominantly from the cold fuel region because of its higher density, 100-300 grams per cubic centimeter compared to the hot spot's 70 grams per cubic centimeter, predicted for an ignited implosion. About 1 to 10 percent of the primary neutrons scatter into the 6-12 MeV range by collisions with background ions as they leave the fuel region, giving a minimum yield required for this measurement of approximately $10^{14}$ neutrons, depending on the details of the density distribution.

The three-dimensional distribution of the primary neutrons, $S_{p}(\mathbf{r}, E)$, is determined from the primary images from all three instruments and the known geometry of the detector system. ${ }^{23,}{ }^{24}$ When solving for $S_{p}(\mathbf{r}, E)$, the DT fuel region is divided into many voxels, typically with dimensions of 2 microns on a side. The same three-dimensional grid is used for determining $\rho(\mathbf{r})$.

To determine $\rho(\mathbf{r})$, three primary assumptions are made. First, it is assumed that the primary neutrons are emitted isotropically. This approximation is good to a few percent. Second, it is assumed that the neutrons only scatter at most once before leaving the fuel region. Third, it is assumed that the fuel region is optically thin to scattered neutrons.

The model used in the density reconstruction is that primary neutrons are emitted from $\mathrm{D}+\mathrm{T}$ reactions in a voxel in all directions. A primary neutron travels through the fuel and has a probability of scattering within any voxel it traverses given by the elastic scattering cross section. The neutron loses energy in the scattering, and its new energy determines the direction it leaves the voxel due to the kinematics of the interaction. ${ }^{19}$, 25 The neutron leaving the fuel is imaged by the pinhole and then recorded at a certain pixel in the detector.

The analysis technique can also be described by starting at the detector and working backward to the fuel. The pinhole and detector pixel determine a subset of voxels that contribute a signal in that pixel. Because the energy of the recorded neutron was selected by gating the detector, the direction from which the primary neutron entered the voxel is determined by the scattering kinematics. To summarize, the geometry of the NIS determines which voxels in the fuel contribute to the signal in the detector. The gating of the detector determines which set of voxels can contribute primary neutrons to the downscattered signal, see Figure 2.

The downscattered signal, $I_{d s}(\mathbf{d}, \widehat{\mathbf{p}})$ recorded in the pixel at position $\mathbf{d}$ on the detector at unit direction $\widehat{\mathbf{p}}$ is the summation of the neutrons scattered by a series of voxels in the fuel region, found by integrating along the line of sight, $\zeta \widehat{\mathbf{p}}$ as shown in Figure 2 which defines the notation and various unit vectors in the laboratory coordinate system. Taking into account that the number of neutrons scattered by a voxel is proportional to the density of the voxel and to the total flux of the primary neutrons through the voxel, this signal can be written as: 


$$
I_{d s}(\mathbf{d}, \widehat{\mathbf{p}})=\int_{-\infty}^{\infty} \rho(\mathbf{d}+\zeta \widehat{\mathbf{p}}) \Phi(\mathbf{d}+\zeta \widehat{\mathbf{p}}, \widehat{\mathbf{p}}) d \zeta
$$

where $\rho$ is the density and the local scattered flux, $\Phi$, which has units of scattered neutrons per unit mass, and is defined by the following equation:

$$
\Phi(\mathbf{r}, \widehat{\mathbf{p}})=\Delta \Omega \bar{A}(\mathbf{r}, \widehat{\mathbf{p}}) \int_{0}^{\infty} \int_{V} S_{p}\left(\mathbf{r}^{\prime}, E\right) \frac{A\left(\mathbf{r}, \mathbf{r}^{\prime}, E\right)}{\left|\mathbf{r}-\mathbf{r}^{\prime}\right|^{2}} \frac{d \mu_{s c}(E, \theta)}{d \Omega} d^{3} r^{\prime} d E
$$

where $\Delta \Omega$ is the acceptance angle of the detector, $\bar{A}(\mathbf{r}, \widehat{\mathbf{p}})$ is the average attenuation factor for scattered neutrons propagating from position $\mathbf{r}$ to the detector, $S_{p}\left(\mathbf{r}^{\prime}, E\right)$ is the distribution of primary neutrons, $A\left(\mathbf{r}, \mathbf{r}^{\prime}, E\right)$ is the attenuation factor for primary neutrons propagating from $\mathbf{r}^{\prime}$ to $\mathbf{r}$, and $\theta=\theta\left(\mathbf{r}, \mathbf{r}^{\prime}, \widehat{\mathbf{p}}\right)$ is the neutron scattering angle in the laboratory coordinate system, i.e. angle between the direction of neutron originated at $\mathbf{r}^{\prime}$ and scattered at $\mathbf{r}$ and projection direction $\widehat{\mathbf{p}}$. The average differential mass scattering coefficient $d \mu_{s c} / d \Omega$ is defined by the following equation:

$$
\frac{d \mu_{s c}(E, \theta)}{d \Omega}=\frac{N_{A}}{m_{a}} \int_{E_{\min }}^{E_{\max }} D\left(E^{\prime}\right) \frac{\partial^{2} \sigma_{s c}\left(E, \theta, E^{\prime}\right)}{\partial \Omega \partial E^{\prime}} \mathrm{d} E^{\prime}
$$

where $N_{A}$ is Avogadro's number, $m_{a}$ is the mean atomic mass of ions composing the plasma, $E_{\min }$ and $E_{\max }$ are the detection energy gates set for scattered neutrons, and the mean double differential scattering cross section, $\partial^{2} \sigma_{s c} / \partial \Omega \partial E$, is the fraction weighted average of the sum of the double differential scattering cross sections, both for elastic and non-elastic scattering processes, of the plasma ions. The relative detector sensitivity, $D\left(E^{\prime}\right)$ is defined as a ratio of the detector signal produced by a neutron with energy $E^{\prime}$ to the signal produced by the DT fusion neutron. ${ }^{18}$ The sensitivity of the specific fiber-optic scintillator used in this neutron imaging system ${ }^{18}$ is approximated in the interval from $1 \mathrm{MeV}$ to 16 $\mathrm{MeV}$ as:

$$
D\left(E^{\prime}\right)=1+\left(\frac{E^{\prime}-E_{0}}{a}\right)-\left(\frac{E^{\prime}-E_{0}}{b}\right)^{2}
$$

with $E^{\prime}$ in units of $\mathrm{MeV}, E_{0}=14.1 \mathrm{MeV}, a=40.32 \mathrm{MeV}$, and $b=17.13 \mathrm{MeV}$. 


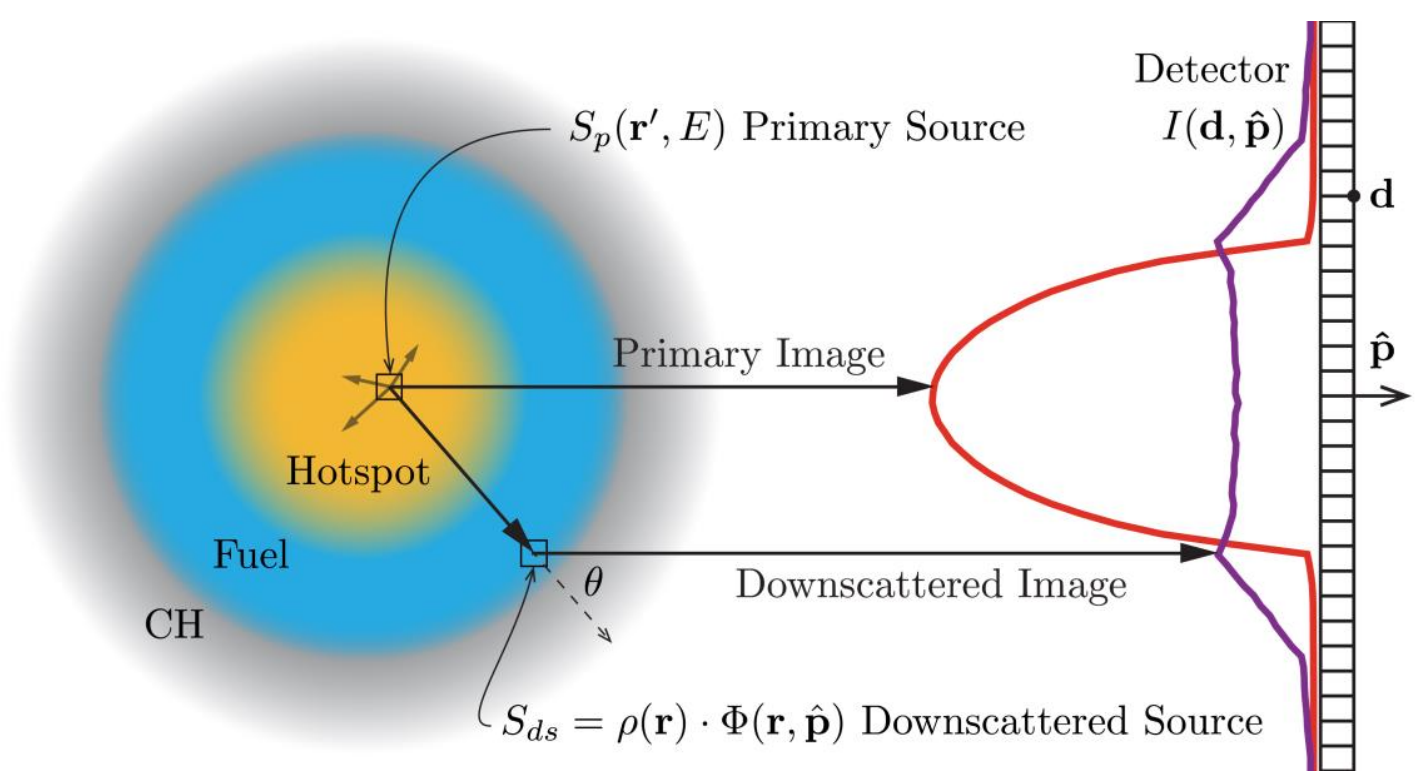

Figure 2. Scattering geometry used in the reconstruction algorithm.

The double differential scattering cross sections are obtained from the ENDF database ${ }^{22}$ after a suitable transformation from ENDF's center-of-mass (CM) reference frame to the laboratory frame used here. For a typical DT plasma, the following scattering processes are included: ${ }^{2} H(n$,elastic $),{ }^{2} H(n, 2 n),{ }^{3} H($ n,elastic $)$. The neutron flux is attenuated as the neutrons traverse the fuel. The primary neutrons are attenuated before they scatter by the factor $A$ and the downscattered neutrons are attenuated by the factor $\bar{A}$. Because the attenuation factors depend on density which is not known a priori, those coefficients need to be estimated before we can compute the local scattering flux $\Phi$. Considering the typical parameters of the ICF implosion - "hot" spot of about 25 microns radius with $25 \mathrm{~g} / \mathrm{cm}^{3}$ density and cold fuel shell of 20 microns thick with $350 \mathrm{~g} / \mathrm{cm}^{3}$ density - we can estimate the attenuation coefficients to be $A \geq \bar{A} \geq 0.8$. Taking into account that the primary source, $S_{p}$, is determined from the primary images which are also attenuated by the factor approximately equal to $A$, it is reasonable to assume that $A=\bar{A}=1$. The consequences of this approximation and approach to overcome those effects are discussed later in Section IV.

With the local scattered flux, $\Phi$, computed and given a set of measured downscattered images, $I_{d s}$, one can solve eq. (1) to determine the density of the fuel assembly.

Ultimately, given a sufficient number of views, this method does not need to assume axial symmetry or any other type of symmetry in order to reconstruct the density distribution. However, very few neutron imaging views are available in inertial confinement fusion experiments. Currently, three primary neutron views and only one downscattered view are available on the National Ignition Facility. The plans are to deploy the second downscattered neutron image line-of-sight in 2020. In this case we need to impose some parameterization of the density distribution to ensure convergence of the solution. Taking into account that the scattered neutron views are orthogonal to the axis of symmetry of the 
NIF system, it is convenient to use Fourier mode decomposition to represent the density distribution:

$$
\rho(\mathbf{r})=\sum_{m=-N}^{N} \rho_{m}(r, z) e^{i m \varphi}
$$

where $(r, \varphi, z)$ are cylindrical coordinates of the vector $\mathbf{r}$. The unknown functions $\rho_{m}(r, z)$ are obtained by substituting the expansion (5) into (1) and solving the resulting equation. The maximum order of the expansion, $N$, is defined by the number of views, so for one view $N=1$, for two views $N=2$, etc. Note that the zero order Fourier mode decomposition is equivalent to Abel inversion. To solve the resulting large system of linear equations we use the Maximization Expectation algorithm with Gibbs prior ${ }^{20}$, which provides a robust solution.

\section{Method Validation using Synthetic Data}

The assumptions of an isotropic primary neutron emission, a single-scattering, and an optically thin material are believed to be reasonable for typical ICF implosions. To demonstrate the fidelity of the presented method and to gauge systematic uncertainties in future physics interpretations based on the density measurement, the method presented here was tested by comparing results from synthetic images prepared from radiationhydrodynamics simulations to the results of the simulations.

First, a static spherically symmetric Monte Carlo $\mathrm{MCNP}^{26}$ simulation is used to validate the single scattering assumption. The synthetic images are produced in MCNP using a perfect pinhole, from a simple geometry: hotspot of 25 -micron radius with $25 \mathrm{~g} / \mathrm{cm}^{3} \mathrm{DT}$, cold fuel shell of 20-micron thickness and $350 \mathrm{~g} / \mathrm{cm}^{3} \mathrm{DT}$, and $\mathrm{CH}$ ablator with density tapering from $150 \mathrm{~g} / \mathrm{cm}^{3}$ to $30 \mathrm{~g} / \mathrm{cm}^{3}$. The geometry of the simulated fuel assembly, the synthetic neutron images and the density reconstruction results are shown in Figure 3. 
(a)

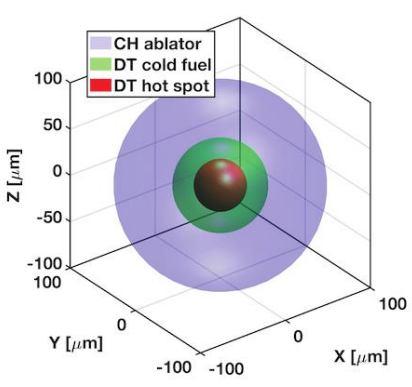

(b)

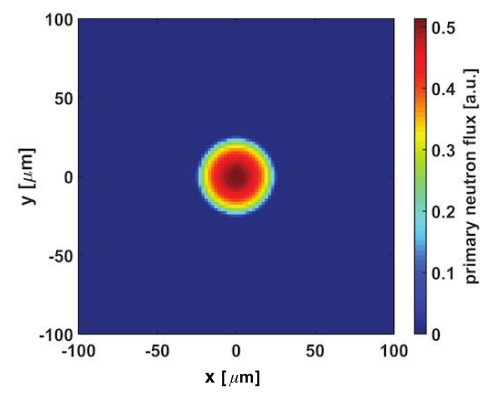

(c)

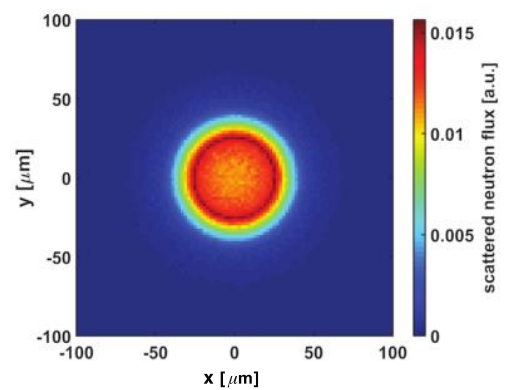

(d)

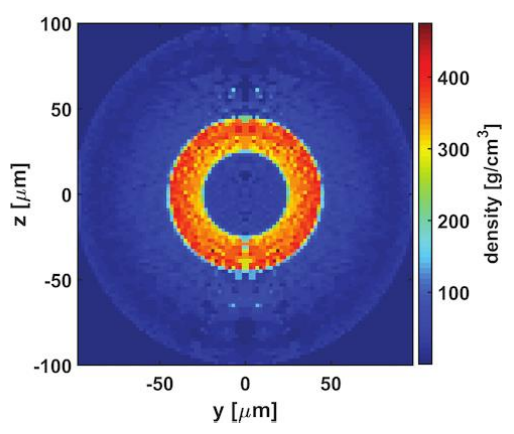

(e)

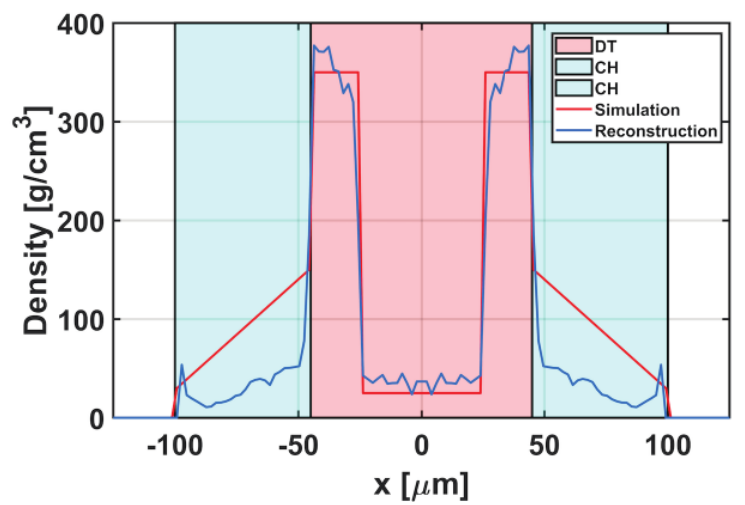

Figure 3. The simple symmetric geometry for the static MCNP simulation contains a DT hot spot, a DT cold fuel and a $\mathrm{CH}$ ablator (a), and produces the resulting synthetic primary (b), and downscattered (c) neutron images. The reconstructed density $(d)$, and a lineout of reconstructed versus simulated density through the center of the source (e) are in good agreement with the original distribution.

As it is evident from the reconstructed density profile (see bottom row in Figure 3 ) the shape, the size, and the average density of the cold fuel shell is well reproduced, however the reconstruction of the density inside the cold fuel shell exhibits a "slope": lower on the inside and higher on the outside. The reconstructed density of the hot spot is approximately $40 \mathrm{~g} / \mathrm{cm}^{3}$ which is higher than original $25 \mathrm{~g} / \mathrm{cm}^{3}$. The detailed analysis taking into account the actual attenuation coefficients $A$ and $\bar{A}$ revealed that both those evident variations of the reconstructed density from the model density are the consequence of neglecting attenuation of the neutrons, i.e. due to the approximation $A=\bar{A}=1$. It is conjectured that this effect could be corrected by the following iterative procedure. After producing an initial density distribution using the method above, calculate new values of the attenuation coefficients, $A$ and $\bar{A}$. These new coefficients would then be inserted into equation (2) and a new value of $\Phi$, the local scattered flux, computed. The new density distribution would then be calculated using the method outlined here. This process could be repeated until the density distribution stops changing by less than a specified value. This iteration should converge rapidly, as the attenuation coefficients both have near unit value, however this conjecture has not yet been tested. 
The current implementation of the technique assumes a constant ion composition over the volume. This assumption results in an incorrect estimation of the ablator density (see Figure 3). However, as it is evident from Figure 3, the size and the shape of the ablator are correctly reproduced. The reduction of ablator density towards to the outer edge of the simulated fuel assembly is also reflected in the reconstruction result.

A more advanced dynamic ICF implosion simulation created by the Chimera ${ }^{27}$ code exhibits a multimode-driven asymmetry. Chimera is a 3-D Eulerian radiation magnetohydrodynamics code developed at Imperial College, London. Synthetic nuclear diagnostic data derived from Chimera include neutron images, time-of-flight spectra, as well as synthetic FNAD data ${ }^{28}$ Using ray-tracing methods, synthetic burn-averaged primary and downscattered neutron images were produced for all three neutron lines of sight currently operating at NIF.

Using the limited view tomography technique described previously, ${ }^{23}$ the three primary images were used to construct a 3-D neutron production volume, which compares well to the simulation truth, especially given the complex shape and small number of views. Figure 4 shows the simulated and reconstructed neutron production volumes.

While the limited-view tomography method does not provide a complete description of internal structure, the overall characteristics are well matched. It is challenging to compare quantitatively 3D volumes, but several metrics show relatively good agreement. At the $20 \%$ maximum emission iso-surface both volumes have an effective radius of 29 microns and contain a volume of $1.05 \cdot 10^{5} \mu \mathrm{m}^{3}$. Figure 5 shows the yield versus volume curves for reconstruction and simulation. For this plot, iso-surface levels are constructed in steps of $5 \%$ of the maximum emission in the volume. Each of these surfaces then contains a specific volume and number of neutrons emitted, which yields the yield versus volume curve. Any deviation from a cubic root curve indicates a deviation from the uniform emission of a sphere. While Figure 5 indicates a slight difference in shape, the volume containing the total yield is the same for reconstruction and simulation. 

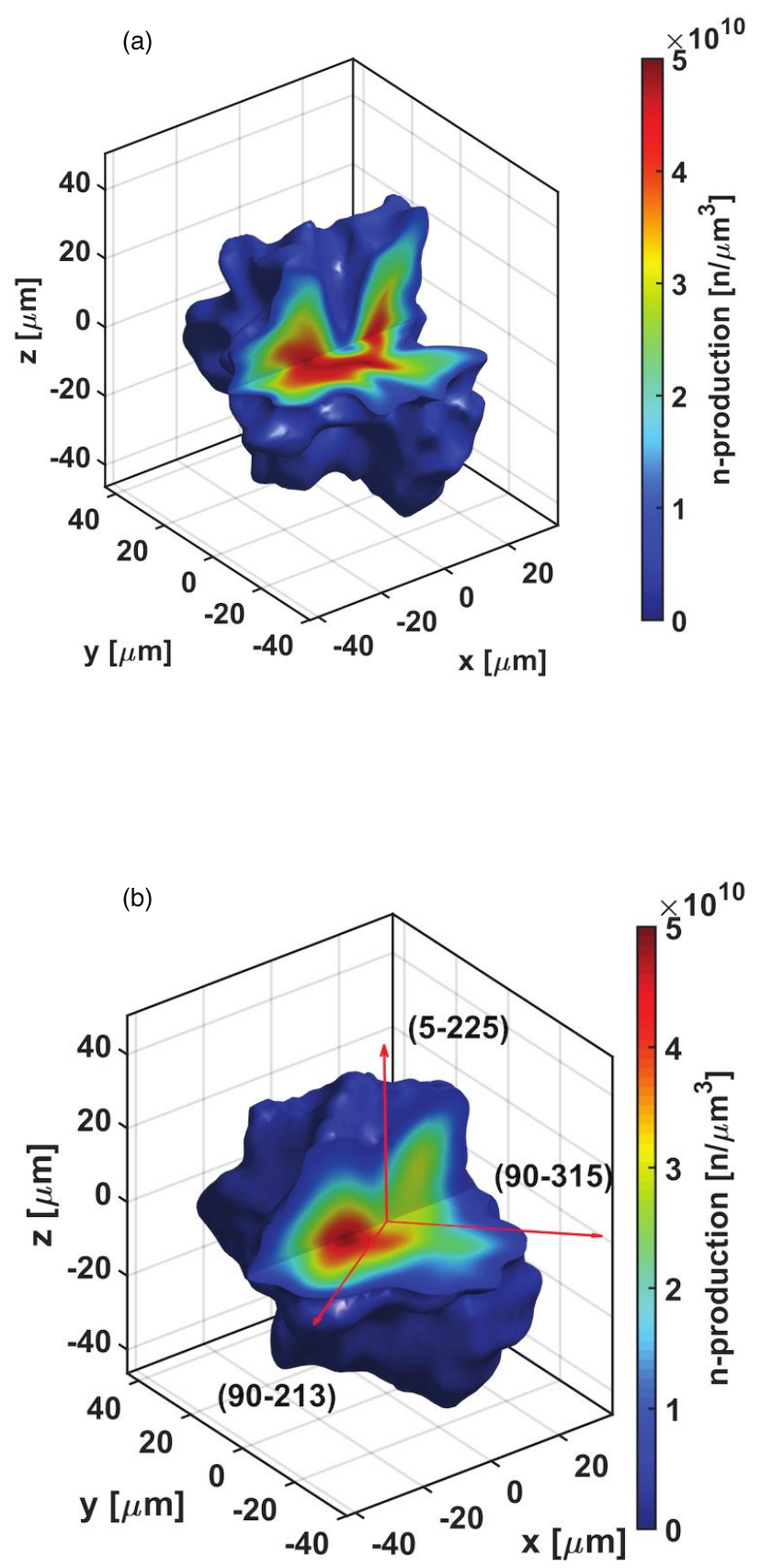

Figure 4. The synthetic (a) and reconstructed (b) neutron production volume of the multi-mode ICF implosion simulated in Chimera. Despite only reconstructing from three views, the shape is well-characterized. 


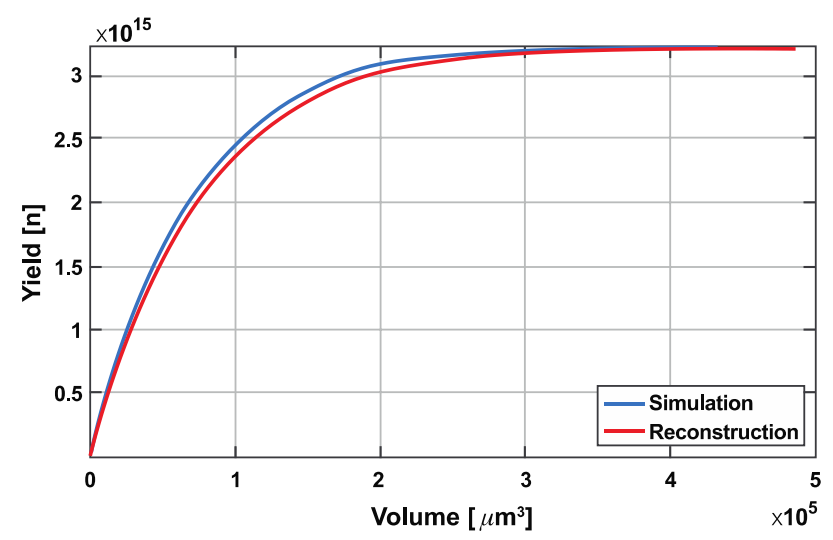

Figure 5. The synthetic versus reconstructed yield vs. volume curves illustrate the quantitative agreement of reconstructed versus simulated burn volumes for a limited-view reconstruction with three lines of sight.

Despite the shortcomings of a reconstruction using only three views, the production yield versus volume is determined adequately (see Figure 5), and the reconstructed production volume serves as the basis for the following density reconstruction from one active line of sight. The density reconstruction result does not change significantly when using the simulated primary source - indicating that the neutron production volume is known sufficiently for this method through the limited-view tomographic reconstruction.

Figure 6 demonstrates that the reconstructed 3-D density distribution from one line of sight using first order Fourier mode decomposition of the density (see eq. (5)) displays the general shape characteristics of the simulation. 
(a)

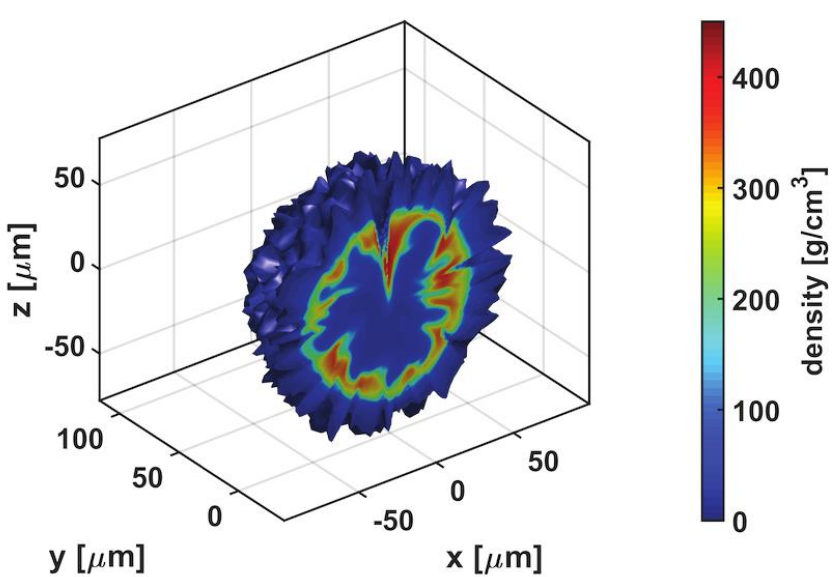

(b)

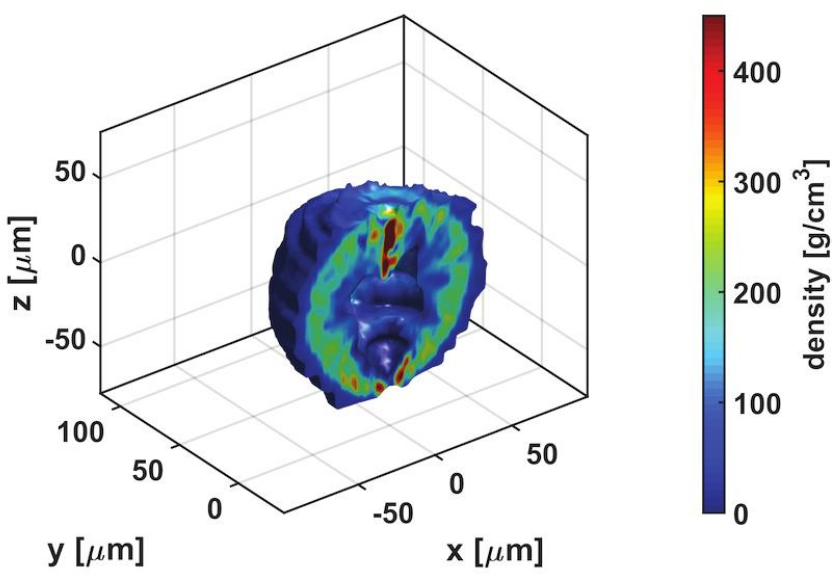

Figure 6. The synthetic (a) and the reconstructed density $(b)$ are in good agreement despite only using one line of sight for the density reconstruction.

The overall mass is underestimated by approximately 20 percent for the burn-averaged case. To study the reconstruction performance for more localized internal structure, we can compare the density profiles along the major coordinate axes, see Figure 7, underlining the complexity of the simulated shape. The reconstruction performs reasonably well, although the high-density region (spike perturbation) along the z-axis as well as more localized smaller high-density features are not well captured. The results are reasonable considering the limited information content from three lines of sight for the neutron production and a single line of sight for the density reconstruction, given also that only a part of the fuel is sampled due to the angular scattering cross sections limiting the view to the front part of the fuel and requiring an assumption of symmetry. The limited-view tomography imposes a resolution effect due to the limited information that can be gained from three and one views; the limited view constrains the ability to reconstruct small-scale variations in 
density. Local density variations can be over- or underestimated up to a factor of two, as seen in Figure 7.

(a)

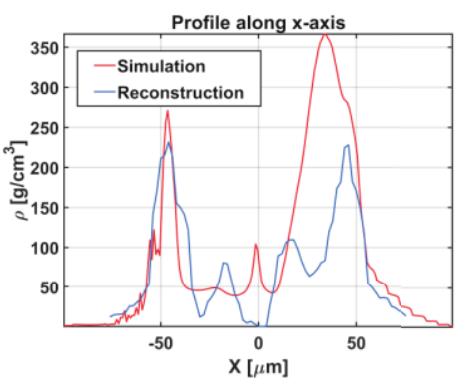

(b)

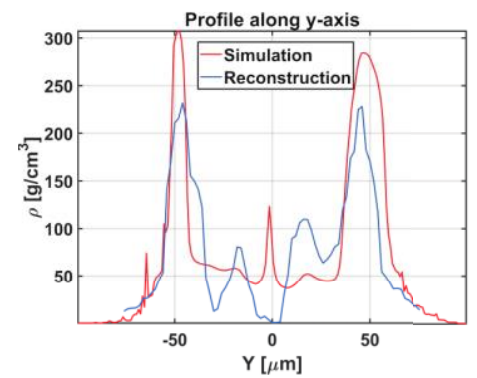

(c)

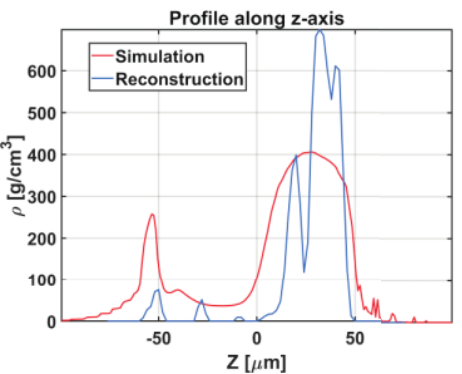

Figure 7. The simulated and reconstructed density profiles along the (a) $X$, (b) $Y$, and (c) $Z$ (vertical) coordinate axes show the reconstruction performs well, given limited information content, but also illustrate challenges along high density regions.

In preliminary studies, the high-density regions are reconstructed more accurately compared to the simulation truth for a single 20-picosecond time slice during the burn time, and the overall mass is then within 10 percent of the simulation. The effect of burnaveraging and possible motion of the hot spot complicate the interpretation of density reconstructions and still need to be examined further; this will be treated in subsequent publications.

The result of applying the density reconstruction method to measured data from NIF shot N190120-001, whose data were shown in Figure 1, is shown in Figure 8. The laser drove the hohlraum with $450 \mathrm{TW}$ peak power and 1.6 MJ. The HDC capsule was 70 microns thick, doped with tungsten, and used a 2-micron-diameter fill tube. The hohlraum was 6.2 $\mathrm{mm}$ in diameter and $11.3 \mathrm{~mm}$ long. 

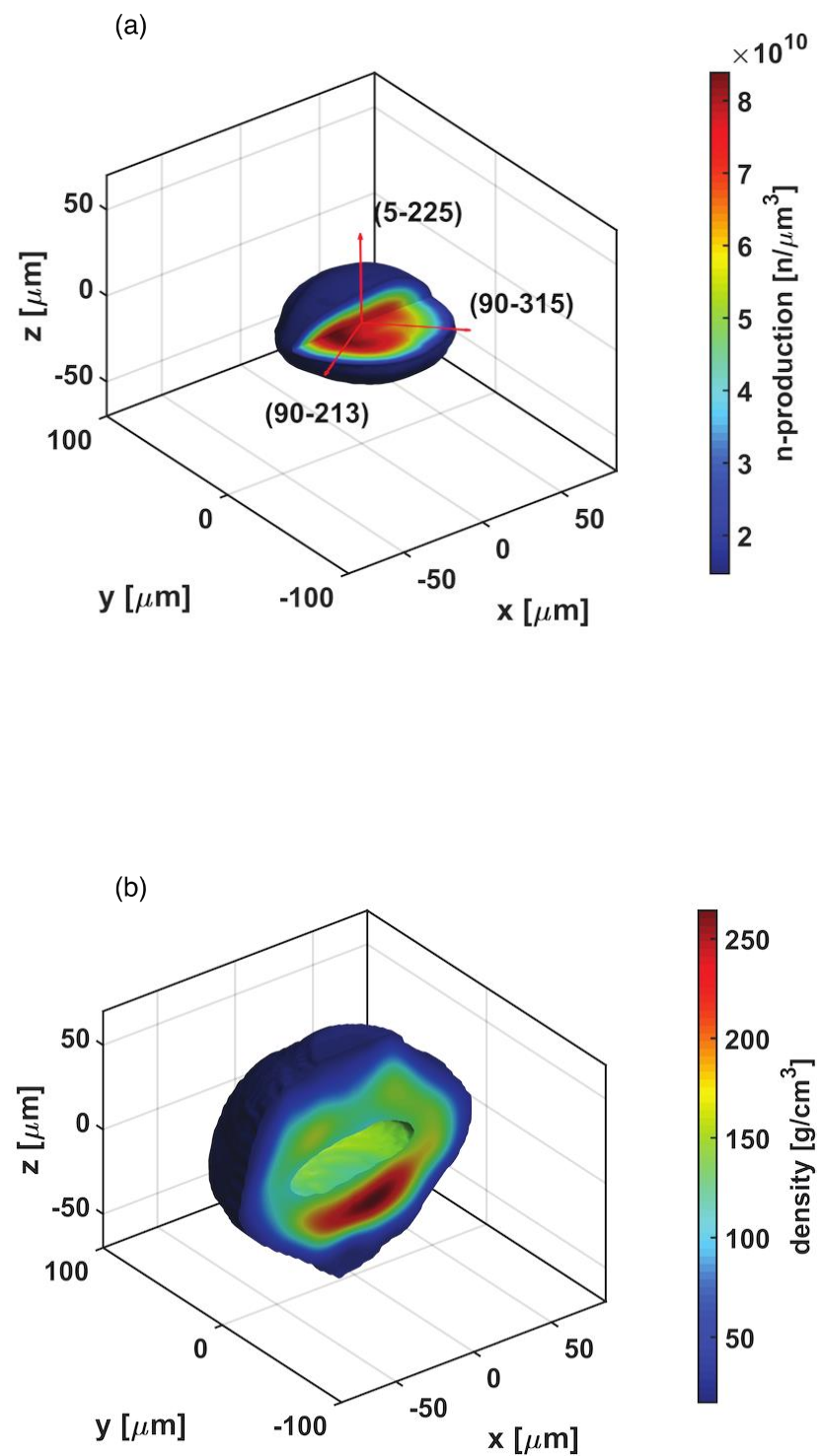

Figure 8. The reconstructed volume neutron production (a) and the reconstructed density with the primary-emission region removed (b) from the measurements in Figure 1 for NIF shot N190120-001. 
(a)

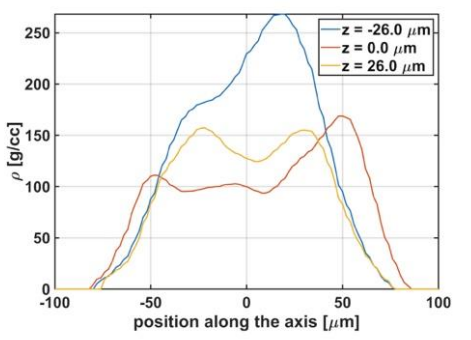

(b)

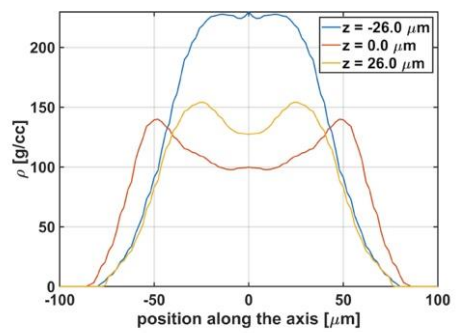

(c)

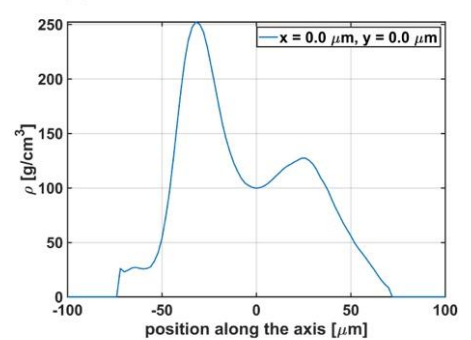

Figure 9. The reconstructed density profiles along (a) axis transverse to the view (90,45), (b) axis along the view (90,315), and (c) vertical axis $(0,0)$ for NIF shot N190120-001. In the equatorial plane, one side of the fuel is twice as dense as the other. Profiles parallel to the equatorial plane show that the asymmetry persists near the edge of the fuel region, which is consistent with a vertical profile in (c)

From the reconstruction, we find that the total reconstructed mass is 141 micrograms out of which 21 micrograms of DT is within the primary-emission region. 
It is evident from Figure 8 that the reconstructed density distribution shows large vertical asymmetry. Because the density reconstruction results using this technique strongly depends on the co-registration of the reconstructed source $S_{p}$ with the downscattered images, due to the term $S_{p}\left(\mathbf{r}^{\prime}, E\right) /\left|\mathbf{r}-\mathbf{r}^{\prime}\right|^{2}$ in eq. (2), the question arises whether this asymmetry due to mis-registration of primary and downscattered images or it reflects the actual density distribution. As was stated before (see Section II) the registration calibration technique ensures that the error in co-registration of the primary and downscattered images does not exceed one micron. The statistical trials simulating co-registration error show that in order to create vertical asymmetry similar to that observed, the co-registration error would need to be about 6 microns, which significantly exceeds the actual uncertainty. This allows us to conclude that the observed vertical asymmetry reflects the actual density distribution.

In addition to vertical asymmetry, one can observe the horizontal asymmetry of the reconstructed density distribution, shown particularly in the equatorial views of Figure 9 where the density is plotted along several lines parallel to the equatorial plane and the vertical axis. This observed assymetry can be related to the fill tube being injected into the fuel during implosion.

\section{Conclusions}

This work describes for the first time a method to determine the density distribution of the DT fuel in an ICF capsule implosion using primary and downscattered neutron images. The single-scattering model is constrained by several assumptions as given in Section III. In theory, these assumptions could be relaxed by more detailed, iterative analysis. However, the accuracy of the current procedure is deemed adequate given the intrinsic spatial resolution of the neutron imaging system. The validation of the reconstruction technique demonstrated in Section IV showed that the novel method is suitable to reconstruct overall density characteristics within the given assumptions.

The current system and method do have limitations. With only one line of sight to the DT core, the current downscattered imaging predominantly samples only the front half of the fuel due to the angular cross sections. Additionally, the present model neglects attenuation and employs a single-scattering approximation. Work is, however, underway to increase the number of lines of sight for downscattered imaging on NIF with novel detectors currently being deployed and designed, and the reconstruction model will be extended to include an iterative approach for attenuation and multiple scattering when more experimental data becomes available.

The NIF neutron images are time integrated over the thermonuclear burn, which is 100$200 \mathrm{psec}$ for typical capsules. If the fuel is moving at $100 \mathrm{~km} / \mathrm{sec}$ as has been measured on some NIF experiments ${ }^{29}$ the motion blur can be as much as 10 microns. No compensation in the data analysis can recover the "non-blurred" density distribution. The authors look forward to compare burn-averaged density results to those from a forthcoming diagnostic currently being developed at NIF - Compton radiography. The technique which has been demonstrated in direct drive experiments ${ }^{30}$ uses a short-pulse laser to create a broad 
spectrum of high-energy $\mathrm{x}$-rays to radiograph the fuel. Compton radiography is a fast 10$100 \mathrm{psec}$ measurement and might allow the study of the above-mentioned motion blur effect of fast-moving hot spots on the burn-averaged density. The two techniques would ideally be applied simultaneously to gain maximum information on the cold fuel. The Compton radiography technique does, however, require that a set of beams be removed from the implosion in order to create the x-rays, which reduces the irradiation symmetry, making it not suitable for all implosions.

Work is currently underway to develop suitable methods and standardized criteria for the community to use effectively the novel density data in comparison with radiationhydrodynamics simulations output. The authors are working with collaborators to identify these comparison criteria, which will aid in drawing further physics conclusions and place reliable uncertainties on additional physical quantities like pressure and temperature extracted from the data.

In summary, this work represents a completely novel diagnostic quantity for inertial confinement implosions at NIF that has never been reported before, and has the potential to have wide-sweeping impact in the field of ICF fusion. The neutron imaging diagnostic now offers the opportunity to fully characterize the fusion fuel averaged over the burntime: the limited-view 3D tomography of the burning hot spot, and the novel information on the absolute density of the cold fuel. With density being one of the primary variables in the criteria for ignition, this data is ideally suited to draw conclusions on fusion performance in comparison to simulations.

Acknowledgements

The authors thank Valerie Fatherley (LANL) for providing information on the detailed configuration of the NIS. The authors also thank the reviewers for their insightful questions that improved this manuscript. Los Alamos National Laboratory, an affirmative action/equal opportunity employer, is operated by Triad National Security, LLC for the National Nuclear Security Administration of U.S. Department of Energy under Contract No. 89233218CNA000001.

\section{References}

1. J. Lindl, Phys. Plasmas 2 (11), 3933-4024 (1995).

2. G. S. Fraley, E. J. Linnebur, R. J. Mason and R. L. Morse, Physics of Fluids 17 (2), 474-489 (1974).

3. B. Cheng, T. J. T. Kwan, Y.-M. Wang and S. H. Batha, Physical Review E 88 (4) (2013).

4. G. P. Grim, R. J. Aragonez, D. P. Atkinson, D. P. Atkinson, S. H. Batha, M. A. Barrios, D. E. Bower, D. K. Bradley, R. A. Buckles, D. D. Clark, D. J. Clark, J. R. Cradick, C. Danly, O. B. Drury, V. E. Fatherley, J. P. Finch, F. P. Garcia, R. A. Gallegos, N. Guler, 
S. M. Glenn, A. H. Hsu, N. Izumi, S. A. Jaramillo, G. A. Kyrala, S. Le Pape, E. N. Loomis, D. Mares, D. D. Martinson, T. Ma, A. J. Mackinnon, F. E. Merrill, G. L. Morgan, C. Munson, T. J. Murphy, P. J. Polk, D. W. Schmidt, R. Tommasini, I. L. Tregillis, A. C. Valdez, P. L. Volegov, T.-S. F. Wang, C. H. Wilde, M. D. Wilke, D. C. Wilson, J. M. Dzenitis, B. Felker, D. N. Fittinghoff, M. Frank, S. N. Liddick, M. J. Moran, G. P. Roberson, P. Weiss, M. I. Kauffman, S. S. Lutz, R. M. Malone and A. Traille, in Ifsa 2011 - Seventh International Conference on Inertial Fusion Sciences and Applications, edited by P. Mora, K. A. Tanaka and E. Moses (2013), Vol. 59.

5. B. Cheng, T. J. T. Kwan, Y. M. Wang, F. E. Merrill, C. J. Cerjan and S. H. Batha, Physics of Plasmas 22 (8) (2015).

6. B. Cheng, T. J. T. Kwan, Y. M. Wang, S. A. Yi, S. H. Batha and F. J. Wysocki, Physics of Plasmas 23 (12) (2016).

7. H. Brysk, Plasma Physics and Controlled Fusion 15 (7), 611-617 (1973).

8. T. J. Murphy, Physics of Plasmas 21 (7) (2014).

9. D. H. Munro, J. E. Field, R. Hatarik, J. L. Peterson, E. P. Hartouni, B. K. Spears and J. D. Kilkenny, Physics of Plasmas 24 (5) (2017).

10. J. D. Kilkenny, P. M. Bell, D. K. Bradley, D. L. Bleuel, J. A. Caggiano, E. L. Dewald, W. W. Hsing, D. H. Kalantar, R. L. Kauffman, D. J. Larson, J. D. Moody, D. H. Schneider, M. B. Schneider, D. A. Shaughnessy, R. T. Shelton, W. Stoeffl, K. Widmann, C. B. Yeamans, S. H. Batha, G. P. Grim, H. W. Herrmann, F. E. Merrill, R. J. Leeper, J. A. Oertel, T. C. Sangster, D. H. Edgell, M. Hohenberger, V. Y. Glebov, S. P. Regan, J. A. Frenje, M. Gatu-Johnson, R. D. Petrasso, H. G. Rinderknecht, A. B. Zylstra, G. W. Cooper and C. Ruiz, Fusion Science and Technology 69 (1), 420-451 (2016).

11. G. Morgan, R. Berggren, P. Bradley, F. Cverna, J. Faulkner, P. Gobby, J. Oertel, F. Swenson, J. Tegtmeier, R. Walton, M. Wilke, D. Wilson and L. Disdier, Rev. Sci. Inst. 72 (1-1), 865-868 (2001).

12. M. D. Wilke, S. H. Batha, P. A. Bradley, R. D. Day, D. D. Clark, V. E. Fatherley, J. P. Finch, R. A. Gallegos, F. P. Garcia, G. P. Grim, S. A. Jaramillo, A. J. Montoya, M. J. Moran, G. L. Morgan, J. A. Oertel, T. A. Ortiz, J. R. Payton, P. Pazuchanics, D. W. Schmidt, A. C. Valdez, C. H. Wilde and D. C. Wilson, Review of Scientific Instruments 79 (10) (2008).

13. F. E. Merrill, D. Bower, R. Buckles, D. D. Clark, C. R. Danly, O. B. Drury, J. M. Dzenitis, V. E. Fatherley, D. N. Fittinghoff, R. Gallegos, G. P. Grim, N. Guler, E. N. Loomis, S. Lutz, R. M. Malone, D. D. Martinson, D. Mares, D. J. Morley, G. L. Morgan, J. A. Oertel, I. L. Tregillis, P. L. Volegov, P. B. Weiss, C. H. Wilde and D. C. Wilson, Review of Scientific Instruments 83 (10) (2012).

14. P. L. Volegov, C. R. Danly, D. N. Fittinghoff, N. Guler, F. E. Merrill and C. H. Wilde, Review of Scientific Instruments 85 (12) (2014).

15. V. E. Fatherley, D. A. Barker, D. N. Fittinghoff, R. L. Hibbard, J. I. Martinez, F. E. Merrill, A. Oertel, D. W. Schmidt, P. L. Volegov and C. H. Wilde, in Target Diagnostics Physics and Engineering for Inertial Confinement Fusion V, edited by J. A. Koch and G. P. Grim (2016), Vol. 9966.

16. V. E. Fatherley, D. A. Barker, D. N. Fittinghoff, R. L. Hibbard, J. I. Martinez, F. E. Merrill, J. A. Oertel, D. W. Schmidt, P. L. Volegov and C. H. Wilde, Review of Scientific Instruments 87 (11) (2016). 
17. V. E. Fatherley, S. H. Batha, C. R. Danly, L. A. Goodwin, H. W. Herrmann, H. J. Jorgenson, J. I. Martinez, F. E. Merrill, J. A. Oertel, D. W. Schmidt, P. L. Volegov, C. A. Wilde, D. N. Fittinghoff, M. J. Ayers, D. A. Barker, G. P. Grim, R. L. Hibbard, N. D. Shingleton and M. A. Vitalich, in Target Diagnostics Physics and Engineering for Inertial Confinement Fusion Vi, edited by J. A. Koch and G. P. Grim (2017), Vol. 10390.

18. C. R. Danly, S. Sjue, C. H. Wilde, F. E. Merrill and R. C. Haight, Review of Scientific Instruments 85 (11) (2014).

19. S. H. Batha, P. L. Volegov, V. E. Fatherley, V. Geppert-Kleinrath and C. H. Wilde, Review of Scientific Instruments 89 (10) (2018).

20. P. Volegov, C. R. Danly, D. N. Fittinghoff, G. P. Grim, N. Guler, N. Izumi, T. Ma, F. E. Merrill, A. L. Warrick, C. H. Wilde and D. C. Wilson, Review of Scientific Instruments 85 (2) (2014).

21. S. Le Pape, L. F. B. Hopkins, L. Divol, A. Pak, E. L. Dewald, S. Bhandarkar, L. R. Bennedetti, T. Bunn, J. Biener, J. Crippen, D. Casey, D. Edgell, D. N. Fittinghoff, M. GatuJohnson, C. Goyon, S. Haan, R. Hatarik, M. Havre, D. D. M. Ho, N. Izumi, J. Jaquez, S. F. Khan, G. A. Kyrala, T. Ma, A. J. Mackinnon, A. G. MacPhee, B. J. MacGowan, N. B. Meezan, J. Milovich, M. Millot, P. Michel, S. R. Nagel, A. Nikroo, P. Patel, J. Ralph, J. S. Ross, N. G. Rice, D. Strozzi, M. Stadermann, P. Volegov, C. Yeamans, C. Weber, C. Wild, D. Callahan and O. A. Hurricane, Physical Review Letters 120 (24) (2018).

22. D. A. Brown, M. B. Chadwick, R. Capote, A. C. Kahler, A. Trkov, M. W. Herman, A. A. Sonzogni, Y. Danon, A. D. Carlson, M. Dunn, D. L. Smith, G. M. Hale, G. Arbanas, R. Arcilla, C. R. Bates, B. Beck, B. Becker, F. Brown, R. J. Casperson, J. Conlin, D. E. Cullen, M. A. Descalle, R. Firestone, T. Gaines, K. H. Guber, A. I. Hawari, J. Holmes, T. D. Johnson, T. Kawano, B. C. Kiedrowski, A. J. Koning, S. Kopecky, L. Leal, J. P. Lestone, C. Lubitz, J. I. Marquez Damian, C. M. Mattoon, E. A. McCutchan, S. Mughabghab, P. Navratil, D. Neudecker, G. P. A. Nobre, G. Noguere, M. Paris, M. T. Pigni, A. J. Plompen, B. Pritychenko, V. G. Pronyaev, D. Roubtsov, D. Rochman, P. Romano, P. Schillebeeckx, S. Simakov, M. Sin, I. Sirakov, B. Sleaford, V. Sobes, E. S. Soukhovitskii, I. Stetcu, P. Talou, I. Thompson, S. van der Marck, L. Welser-Sherrill, D. Wiarda, M. White, J. L. Wormald, R. Q. Wright, M. Zerkle, G. Zerovnik and Y. Zhu, Nuclear Data Sheets 148, 1142 (2018).

23. P. L. Volegov, C. R. Danly, F. E. Merrill, R. Simpson and C. H. Wilde, Journal of Applied Physics 118 (20) (2015).

24. P. L. Volegov, C. R. Danly, D. Fittinghoff, V. Geppert-Kleinrath, G. Grim, F. E. Merrill and C. H. Wilde, Journal of Applied Physics 122 (17) (2017).

25. A. K. Ghatak, American Journal of Physics 32 (2), 163-\& (1964).

26. C. J. Werner (Editor), Los Alamos National Laboratory, LA-UR-17-29981 (2017).

27. J. P. Chittenden, B. D. Appelbe, F. Manke, K. McGlinchey and N. P. L. Niasse, Physics of Plasmas 23 (5) (2016).

28. A. J. Crilly, B. D. Appelbe, K. McGlinchey, C. A. Walsh, J. K. Tong, A. B. Boxall and J. P. Chittenden, Physics of Plasmas 25 (12) (2018).

29. R. Hatarik, R. C. Nora, B. K. Spears, M. J. Eckart, G. P. Grim, E. P. Hartouni, A. S. Moore and D. J. Schlossberg, Review of Scientific Instruments 89 (10) (2018).

30. R. Tommasini, S. P. Hatchett, D. S. Hey, C. Iglesias, N. Izumi, J. A. Koch, O. L. Landen, A. J. MacKinnon, C. Sorce, J. A. Delettrez, V. Y. Glebov, T. C. Sangster and C. Stoeckl, Physics of Plasmas 18 (5) (2011). 


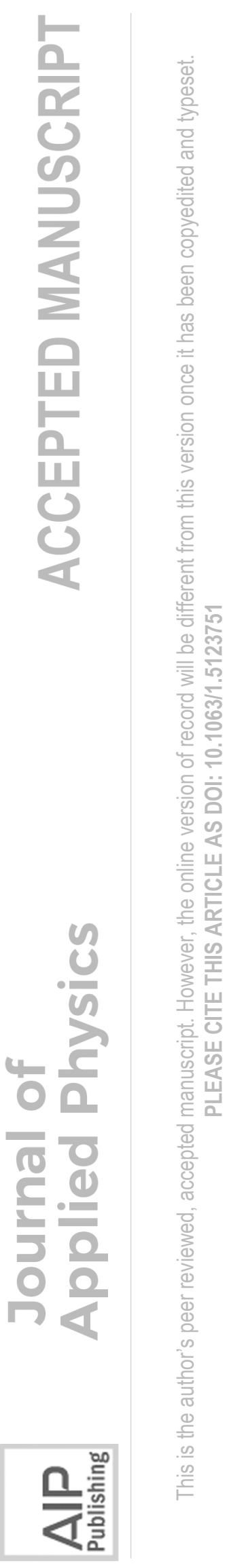


(a)

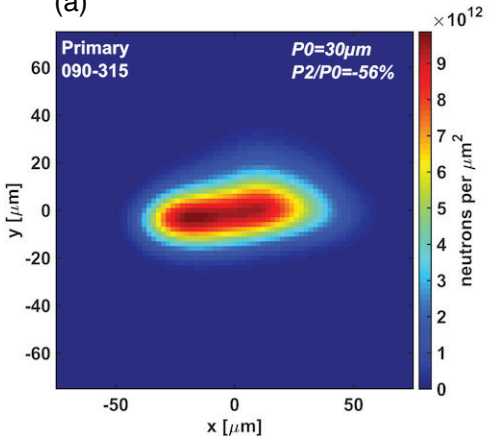

(d)

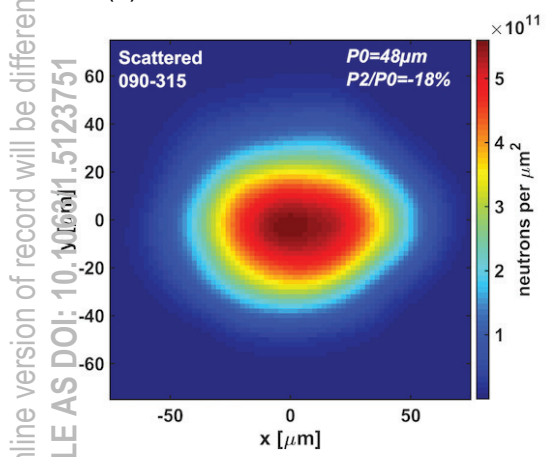

(b)

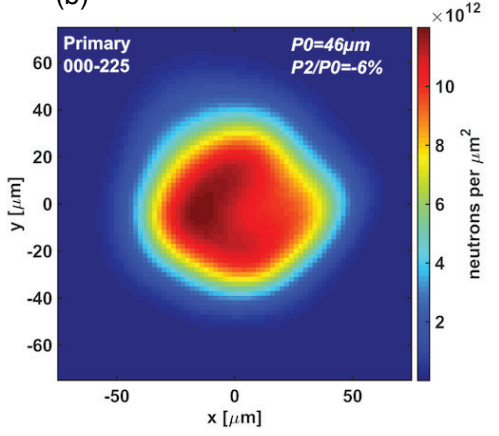

(e)

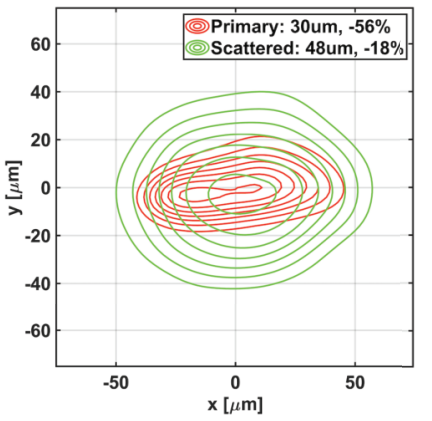

(c)

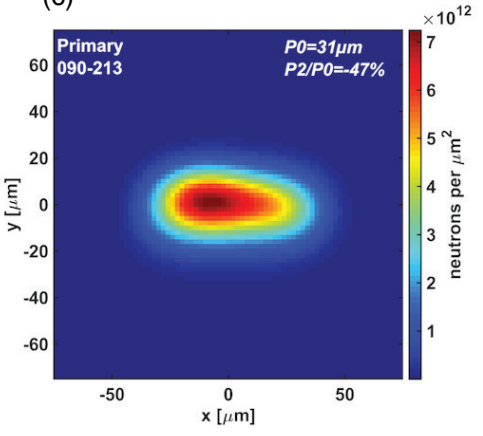

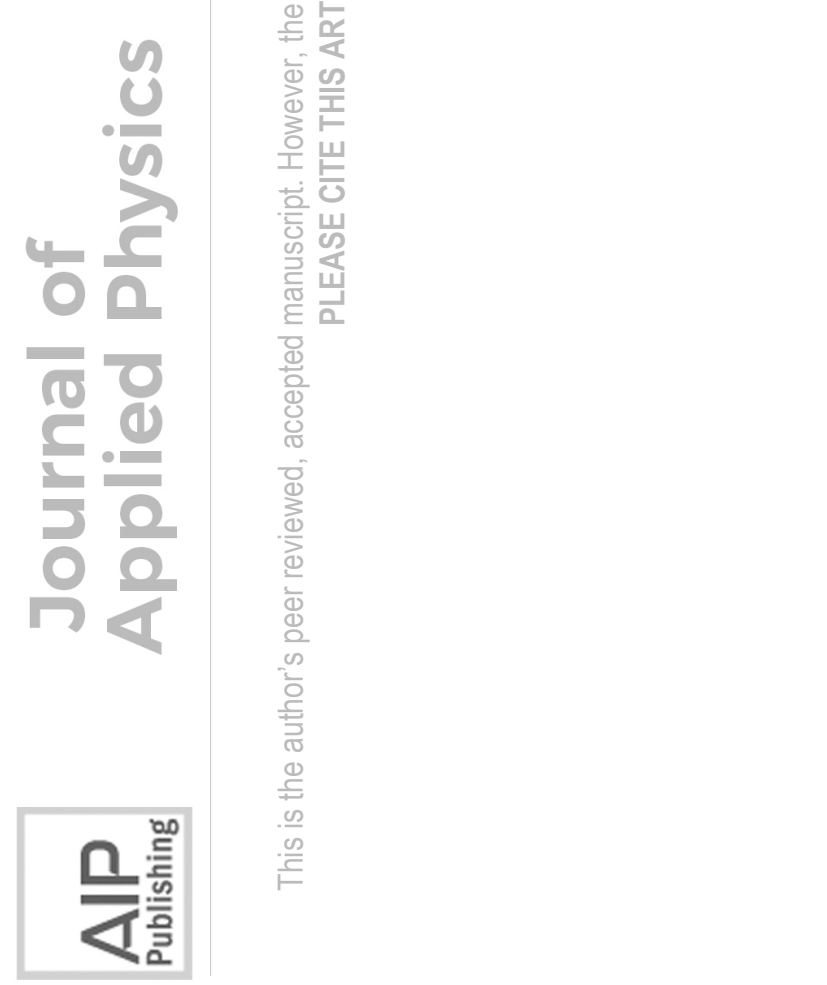




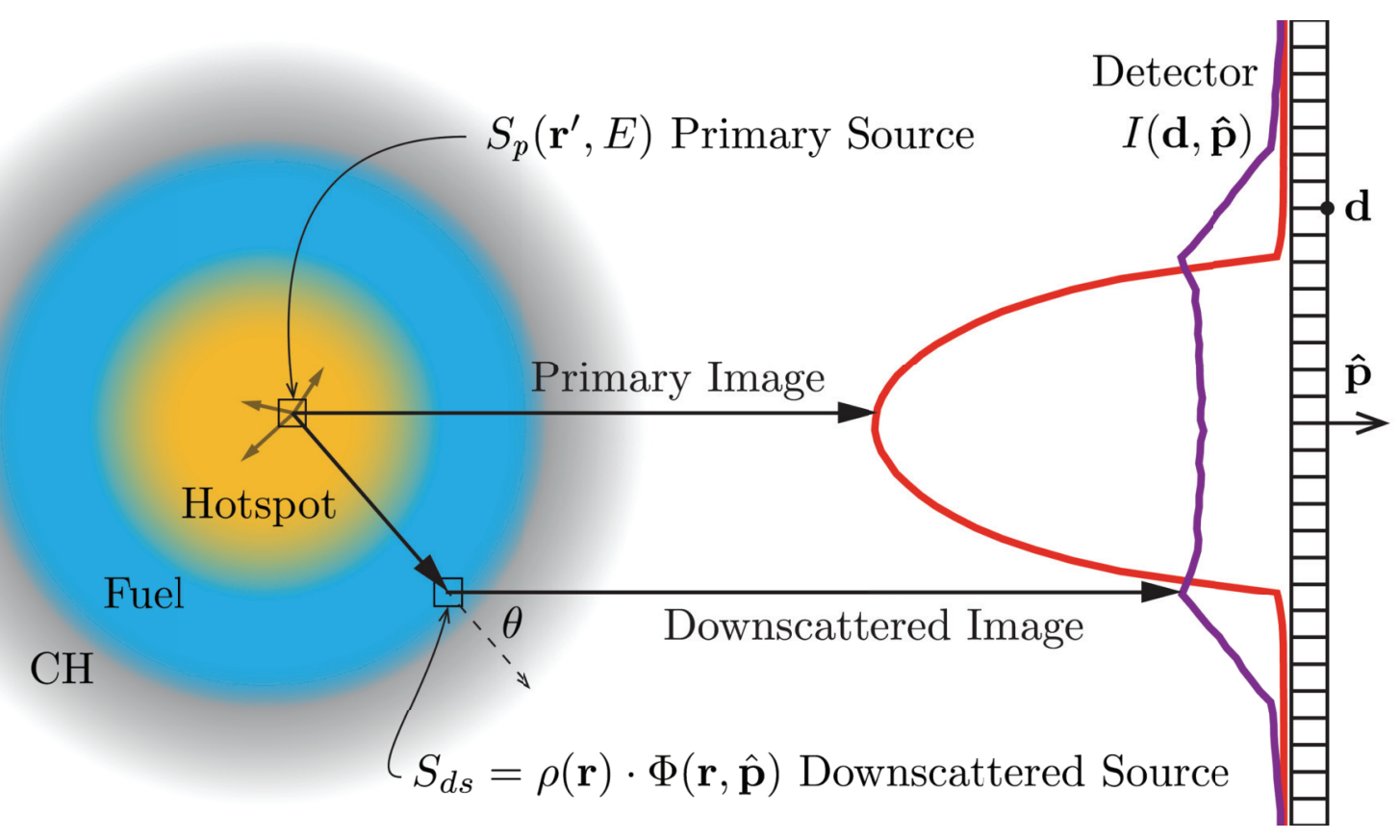


(a)

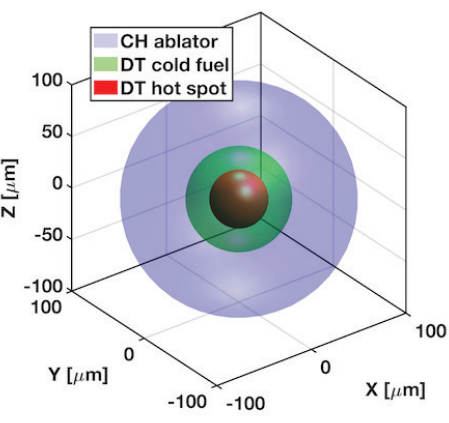

(d)

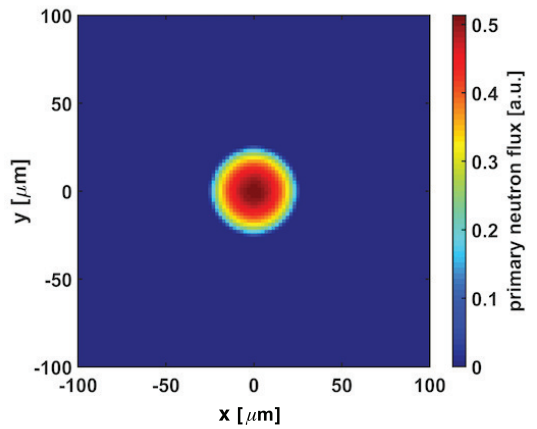

(e) (c)

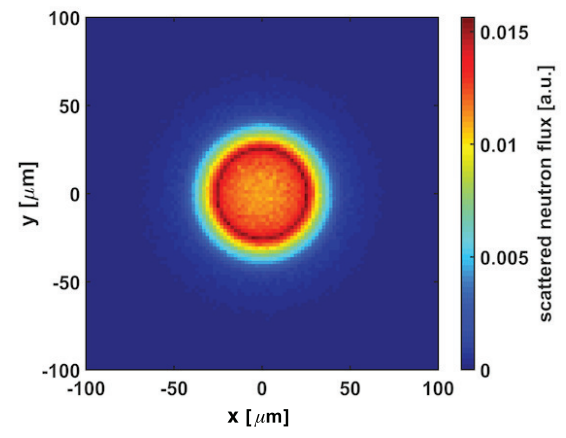

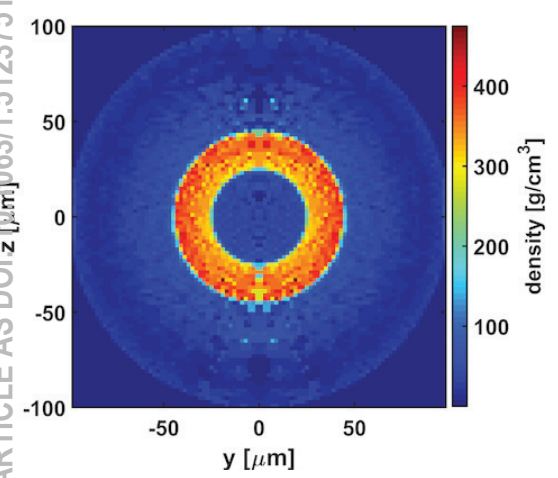

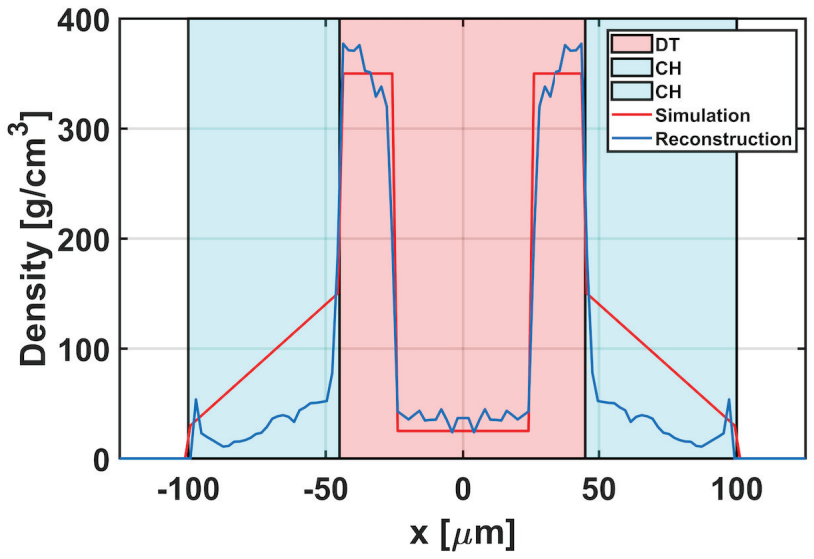



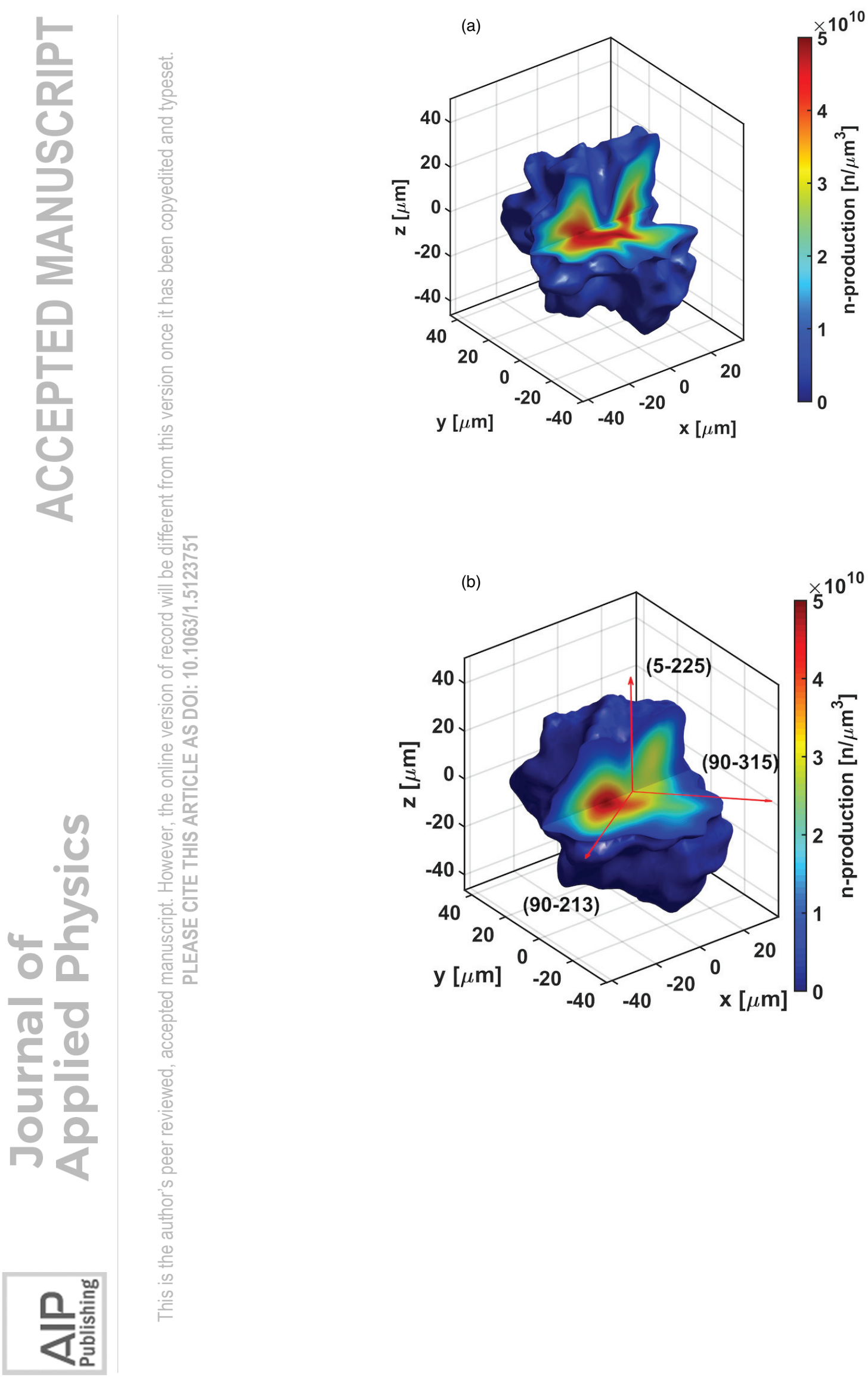

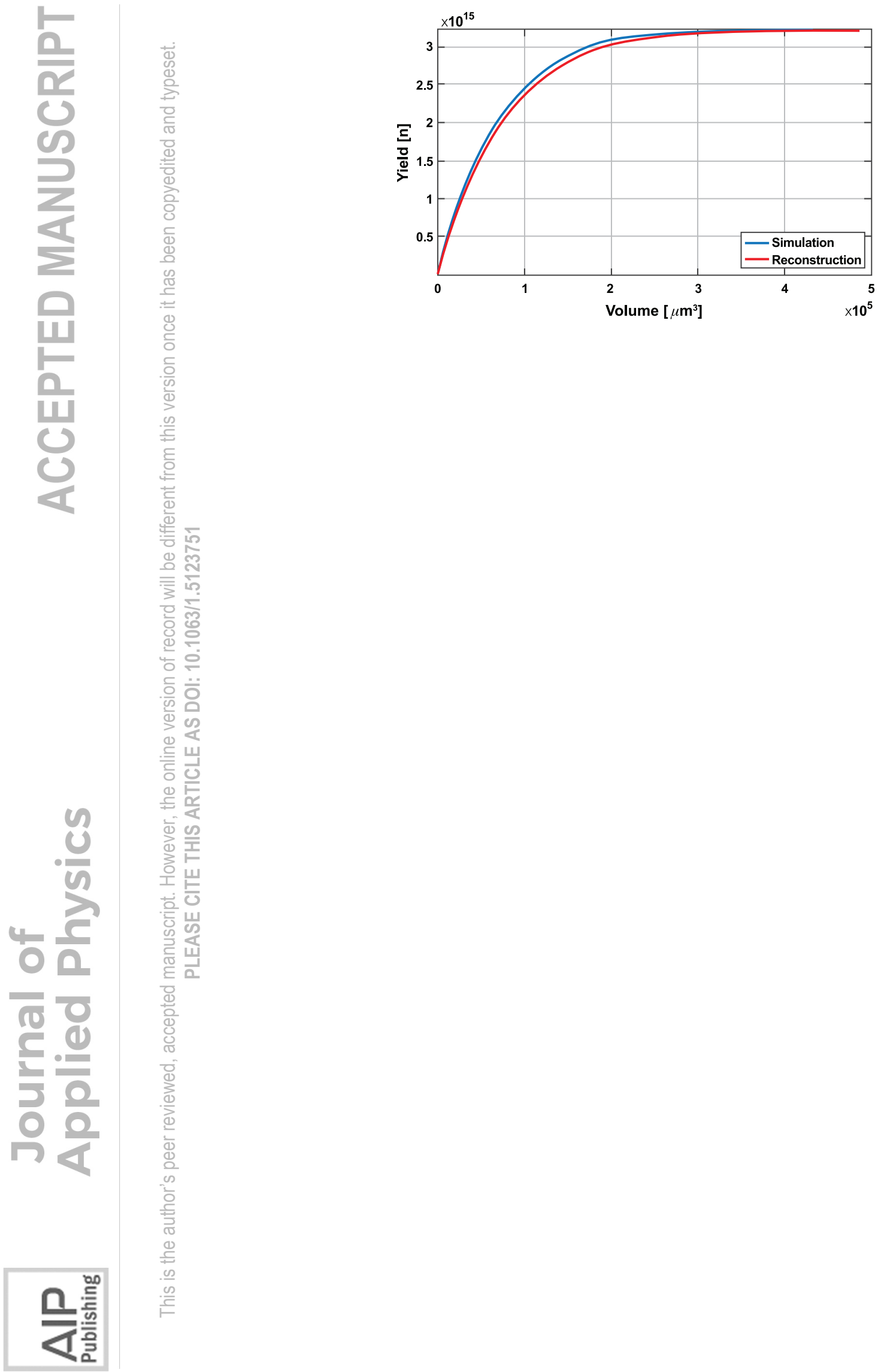
(a)

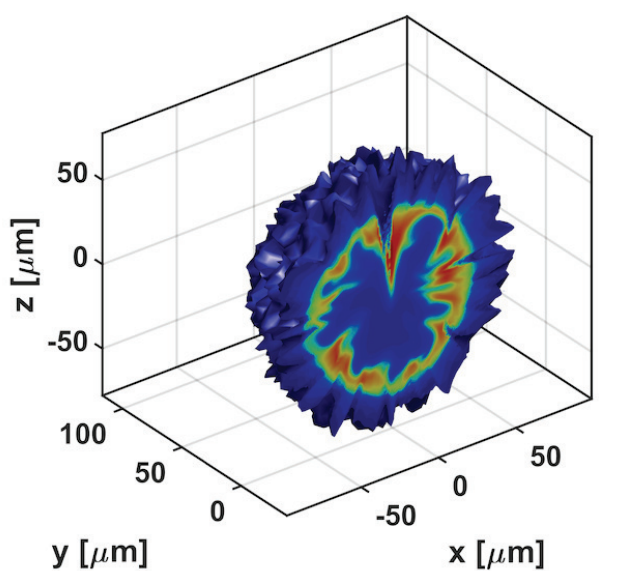

(b)

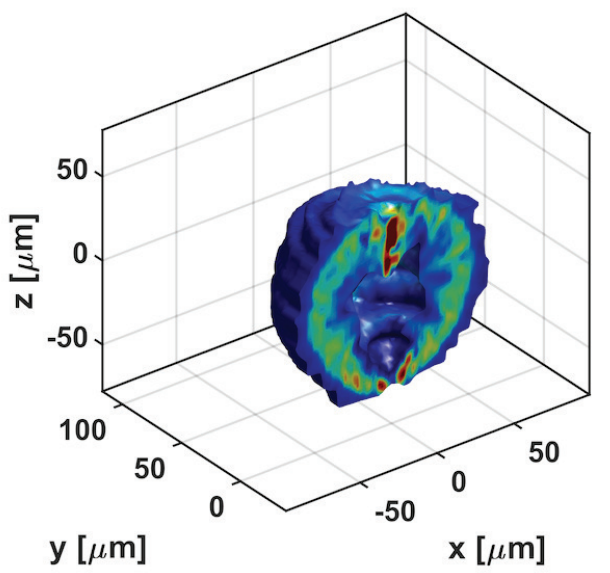

$-400$ 
(a)

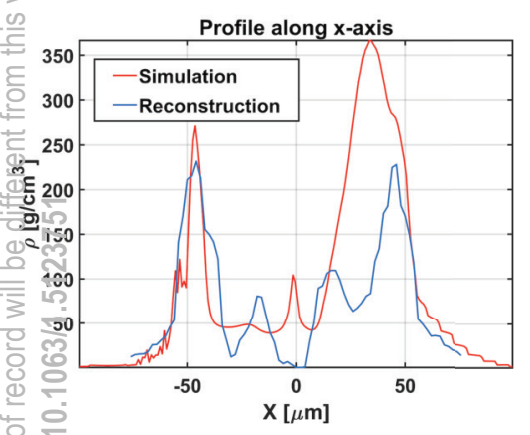

(b)

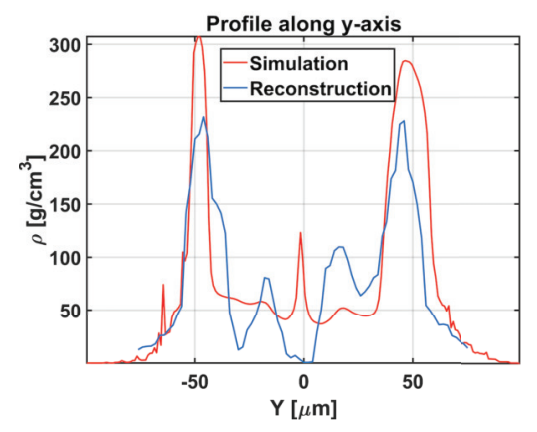

(c)

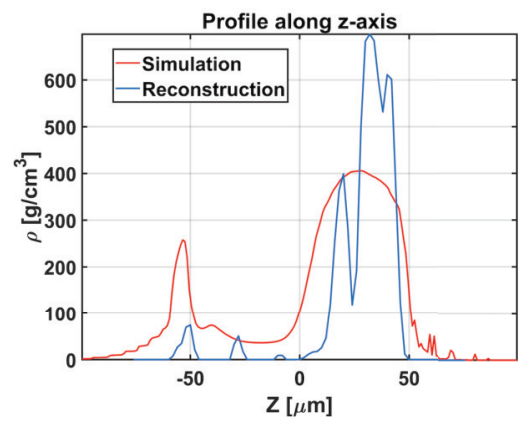

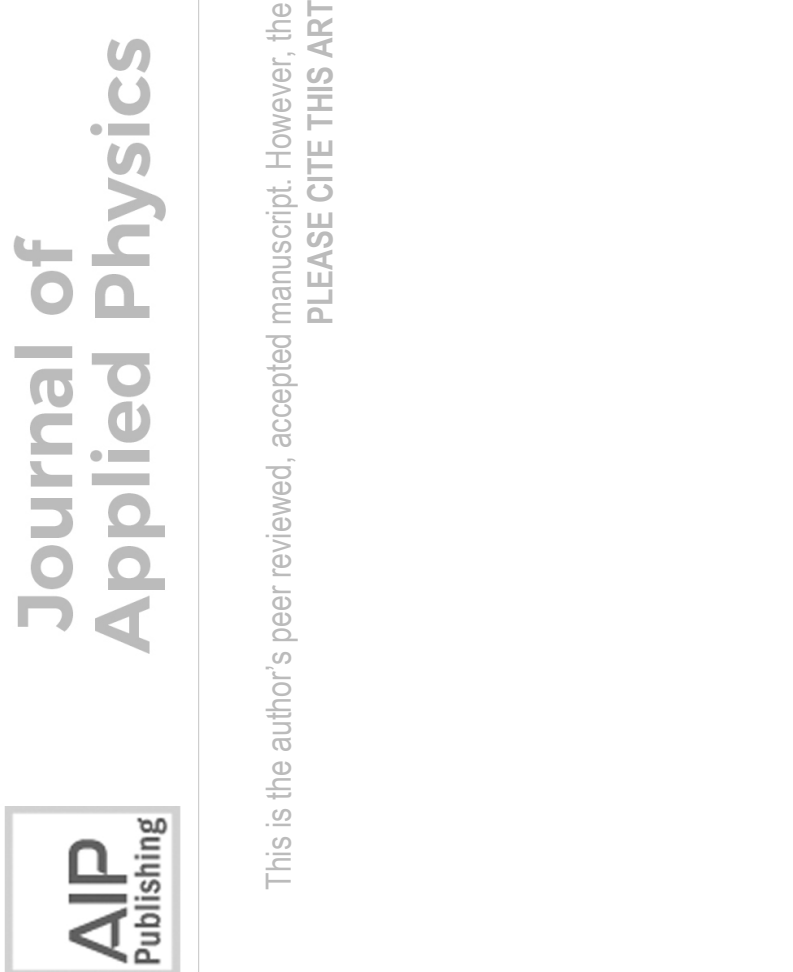



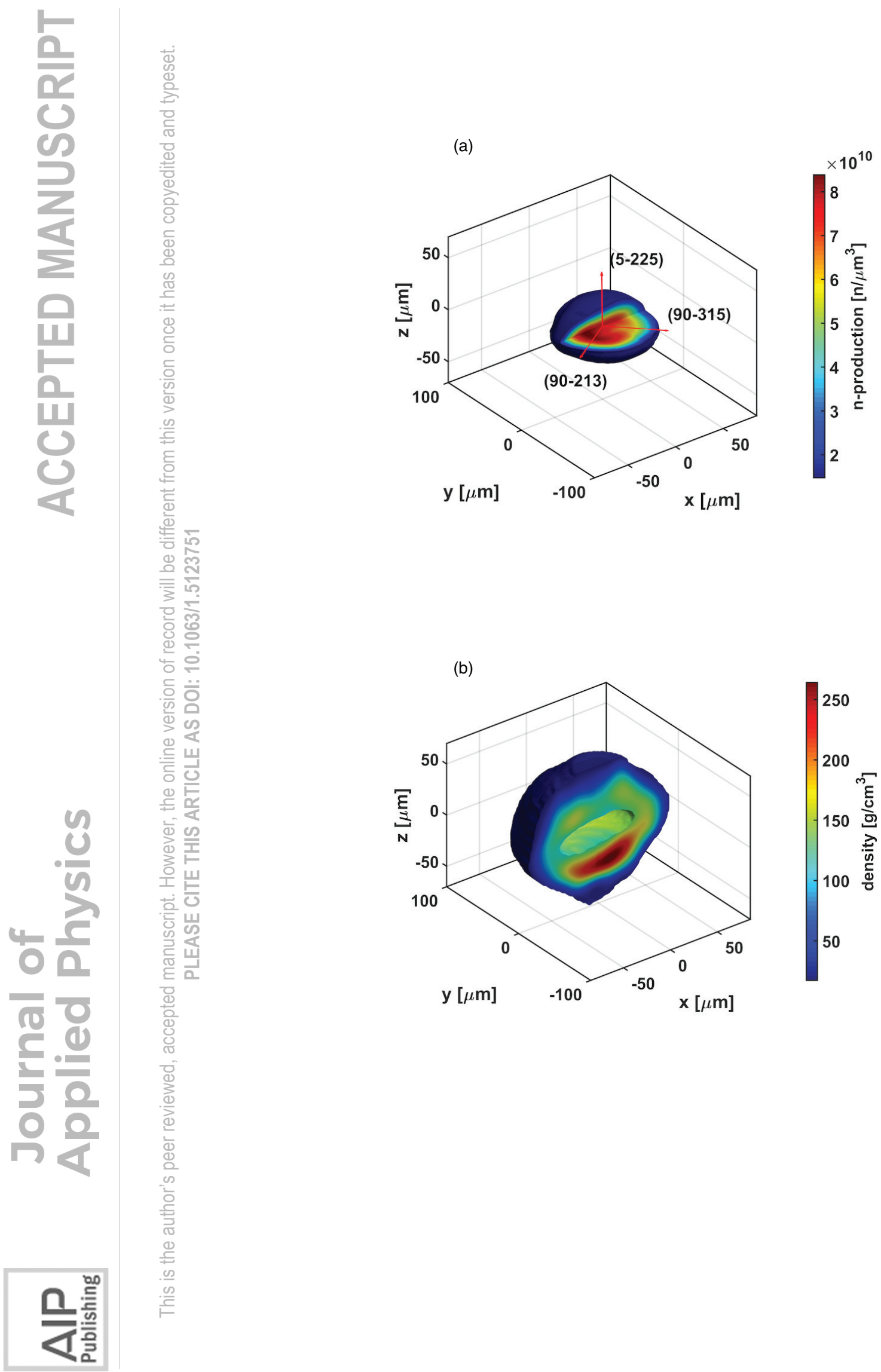

(b)

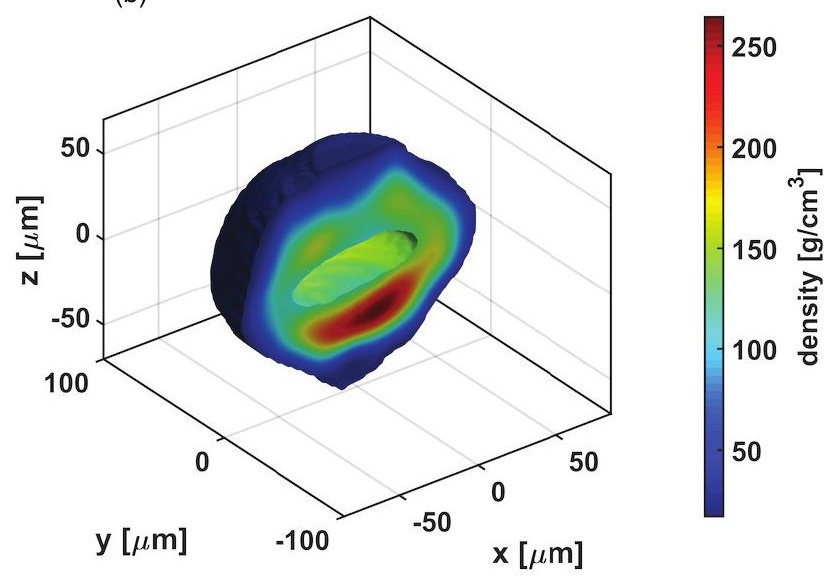


(a)

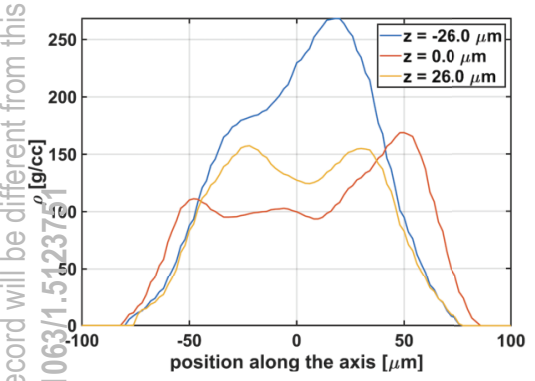

प으.

"흐을

듬

닌

电

플

든

딸 만

뜨을

함

오쁠

드이

을

号

है एँ

( 1

$\begin{array}{ll}10 \\ 0 \\ 0 & 0 \\ 0 & 0 \\ 0 & 0\end{array}$ (b)

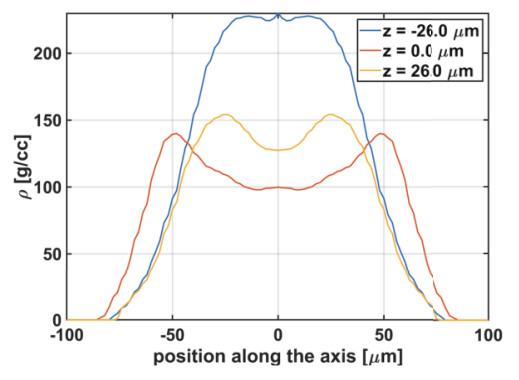

(c)

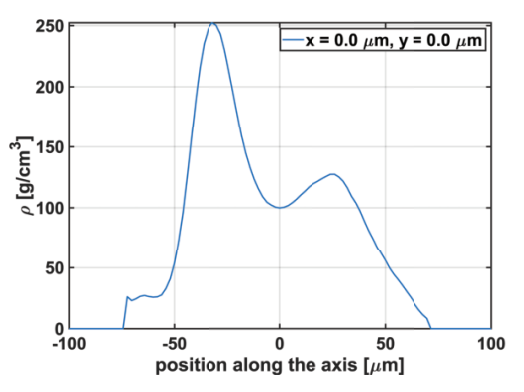

